\title{
Microbiota induces tonic CCL2 systemic levels that control pDC trafficking in steady state
}

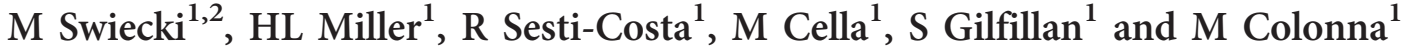

Plasmacytoid dendritic cells (pDCs) detect viruses initiating antiviral type I interferon responses. The microbiota is known to shape immune responses, but whether it influences $\mathrm{PDC}$ homeostasis and/or function is poorly understood. By comparing pDCs in germ-free and specific pathogen-free mice, we found that the microbiota supports homeostatic trafficking by eliciting constitutive levels of the chemokine CCL2 that engages CCR2. Mononuclear phagocytes were required for tonic CCL2 levels. CCL2 was particularly important for trafficking of a CCR2 ${ }^{\text {hi }}$ subset of pDCs that produced proinflammatory cytokines and was prone to apoptosis. We further demonstrated that CCR2 was also essential for pDC migration during inflammation. Wild-type (WT):Ccr2 ${ }^{--}$mixed bone marrow chimeras revealed that CCR2 promotes pDC migration in a cell-intrinsic manner. Overall, we identify a novel role for the microbiota in shaping immunity, which includes induction of CCL2 levels that control homeostatic trafficking of pDCs.

\section{INTRODUCTION}

Plasmacytoid dendritic cells (pDCs) are a subset of DCs recognized for their ability to detect viral genomes through Toll-like receptor-7 and Toll-like receptor-9, and secrete large quantities of type I interferon (IFN-I), promoting early antiviral defense. ${ }^{1-3}$ Unstimulated pDCs also contribute to immunological tolerance in steady state through multiple mechanisms. In contrast, inappropriately activated $\mathrm{pDCs}$ have been implicated in the pathogenesis of autoimmune diseases characterized by a IFN-I signature such as systemic lupus erythematosus. ${ }^{2,4}$

pDCs have unique trafficking properties compared with conventional DCs. ${ }^{2,5} \mathrm{pDCs}$ are generated in the bone marrow (BM), circulate in the blood, and reach lymphoid organs and non-lymphoid tissues in steady state. During inflammation, pDCs are part of the inflammatory infiltrate that extravasates from the blood vessels into sites of tissue damage and draining lymph nodes (LNs). pDC migration is controlled by multiple chemokine receptors and homing molecules. In steady state, CCR5 and, to a lesser extent, CCR2 promote pDC egress from the $\mathrm{BM},{ }^{6}$ while $\mathrm{CD} 62 \mathrm{~L}, \mathrm{CCR} 7$, and CXCR4 mediate migration of pDCs into peripheral LNs and the splenic white pulp. ${ }^{2,5}$ CCR9 and its ligand CCL25 drive pDC migration to the thymus, where they transport peripheral antigens and induce tolerance; ${ }^{7}$ CCR9 is also important for $\mathrm{pDC}$ recruitment to the small intestine. ${ }^{8}$ Recently, it was shown that pDCs express MAdCAM- 1 and $\beta 7$ integrin, which promote their trafficking into the intestinal intraepithelial compartment. ${ }^{9}$ During inflammation, the ligands for E-selectin, $\beta 1$ and $\beta 2$ integrins, CXCR3, CCR5, CCR7, and the chemerin receptor ChemR23 drive $\mathrm{pDC}$ migration to sites of inflammation and draining LNs. ${ }^{2,5}$ A subset of human tonsil pDCs express CCR6 and CCR10, which enable them to migrate to inflamed epithelia in response to CCL20 and CCL27. ${ }^{10}$ In addition, pDCs have functional receptors for adenosine, $\mathrm{C} 3 \mathrm{a}$ and $\mathrm{C} 5 \mathrm{a}$, which are released by damaged tissues. ${ }^{5}$ Thus, a complex network of chemokines enables pDC trafficking in steady state and during disease.

The microbiota shapes mucosal and systemic immune responses by promoting homeostasis and function of many immune cell types including monocytes, granulocytes, DCs, T cells and $\mathrm{B}$ cells. ${ }^{11-18}$ However, whether and how the microbiota influences $\mathrm{pDC}$ homeostasis and functions remains largely unexplored. One study found that $\mathrm{pDC}$ frequencies and numbers were largely intact in germ-free (GF) mice, whereas mice bearing restricted enteric microbial communities had a dramatic reduction of pDCs in the spleen and mesenteric LN $(\mathrm{MLN}),{ }^{19}$ suggesting a potential role for specific microbial

${ }^{1}$ Department of Pathology and Immunology, Washington University School of Medicine, St Louis, Missouri, USA and ${ }^{2}$ Janssen Research and Development LLC, Spring House, Pennsylvania, USA. Correspondence: M Colonna (mcolonna@pathology.wustl.edu) 
communities in influencing pDC development and/or distribution. Moreover, commensal microbial products, such as polysaccharide $\mathrm{A}$, influence $\mathrm{pDC}$ functions, endowing them with the capacity to promote the differentiation of interleukin10 producing regulatory T cells. ${ }^{20}$ Gut-associated pDCs are also capable of generating mucosal B-cell responses, ${ }^{21,22}$ promoting oral tolerance ${ }^{23}$ and may influence Th17 generation. ${ }^{24}$

Here we analysed pDCs in GF and specific pathogen-free (SPF) mice, and found that the microbiota was essential to sustain constitutive levels of CCL2 that promoted maintenance of pDCs in blood and peripheral tissues. Examination of pDC subsets revealed that in SPF mice the ratio between CCR $2^{\text {lo }}$ and CCR $2^{\text {hi }}$ pDCs was $\sim 2: 1$ in the BM and 1:1 in the periphery, whereas in GF mice the ratio was close to $2: 1$ also in the periphery, indicating a selective reduction of peripheral CCR 2 hi pDCs. Functionally, CCR2 ${ }^{\text {hi }}$ pDCs were more prone to proinflammatory cytokine production, as well as spontaneous and glucocorticoid-induced apoptosis, compared to CCR $2^{\text {lo }}$ pDCs. GF mice also had reduced numbers of circulating Ly6C ${ }^{\text {hi }} \mathrm{CD} 115^{+}$inflammatory monocytes, which depend on CCR2 to exit the $\mathrm{BM},{ }^{25-27}$ thus supporting a general role for microbiota in the migration of CCR2 ${ }^{+}$cells in steady state. The cell-intrinsic requirement for CCR2-CCL2 signaling for $\mathrm{pDC}$ trafficking in steady state was confirmed using WT:Ccr $2^{-/-} \mathrm{BM}$ chimeras. Depletion experiments with clodronate (Clod) liposomes and anti-CSF-1R monoclonal antibody (mAb) revealed that mononuclear phagocytes were at least in part required for the production of CCR2 ligands and $\mathrm{pDC}$ recruitment to $\mathrm{LN}$ in steady state. We further demonstrated that CCR2 was essential for pDC migration under inflammatory conditions. Taken together, our findings suggest a role of microbiota in inducing steady-state levels of CCL2, which is produced at least in part by mononuclear phagocytes and engenders homeostatic circulation of pDCs and inflammatory monocytes.

\section{RESULTS}

\section{Microbiota is required for homeostatic pDC distribution}

To address the impact of microbiota on pDC homeostasis, we compared $\mathrm{pDC}$ frequencies and numbers in naive SPF and GF mice. In steady state, peripheral blood pDCs were reduced in frequency and number in GF mice (Figure 1a). Peripheral blood monocytes were also markedly reduced (Figure 1a). Spleen, MLN, small intestine intraepithelial lymphocytes and thymus from GF mice also revealed a reduction in pDCs (Figure $\mathbf{1 b}$ and $\mathbf{c}$ ). To determine whether the defect in peripheral pDCs in GF mice was due to defective generation of pDCs in BM, we measured frequencies and numbers of mature and immature pDCs based on expression of Ly49Q and SiglecH. GF mice showed higher frequencies of mature pDCs $\left(\mathrm{Ly}_{49} \mathrm{Q}^{\text {hi }}\right.$ SiglecH $\left.{ }^{+}\right)$, immature pDCs $\left(\mathrm{Ly} 49 \mathrm{Q}^{\text {lo }}\right.$ SiglecH $\left.{ }^{+}\right)$, and pDC progenitors $\left(\mathrm{Ly}_{49} \mathrm{Q}^{-}\right.$SiglecH $\left.{ }^{+}\right)$, and higher absolute numbers of mature pDCs than SPF mice in the BM (Figure 1d and data not shown). Thus, these data suggested that the microbiota may promote egress of pDCs from BM. a

Peripheral blood
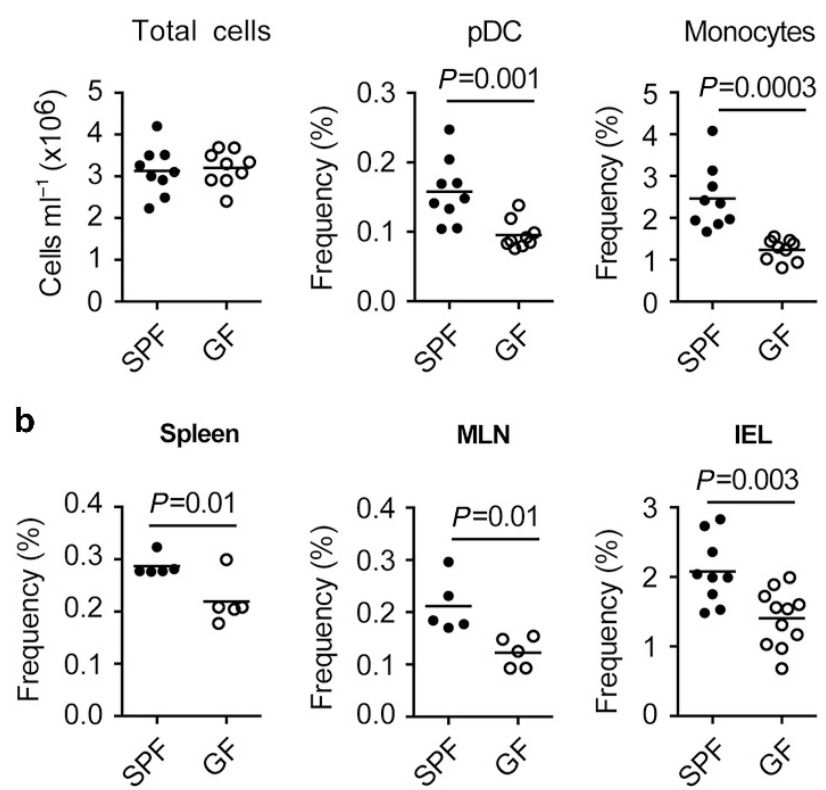

C
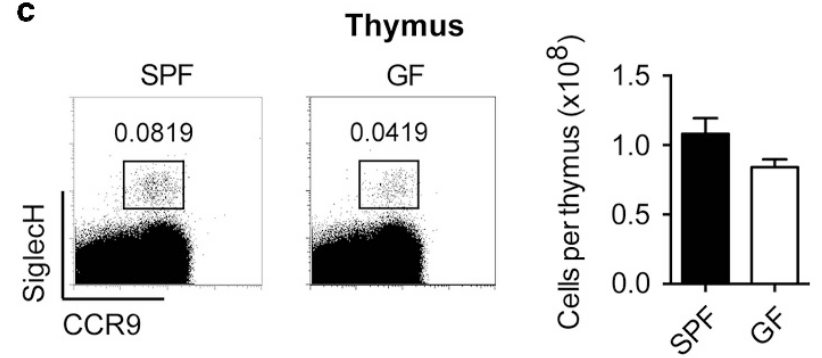

d

Bone marrow
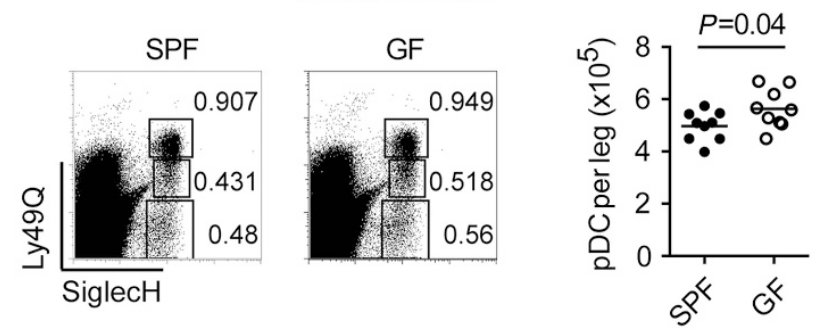

Figure 1 Plasmacytoid dendritic cells (pDCs) are reduced in the periphery of germ-free (GF) mice and accumulate in the bone marrow (BM). (a) Peripheral blood from specific pathogen-free (SPF) and GF mice was analyzed for total cellularity and frequencies of pDCs $\left(\right.$ SiglecH $\left.{ }^{+} \mathrm{BST}^{+}\right)$and monocytes $\left(\mathrm{CD} 11 \mathrm{~b}^{+} \mathrm{Ly}_{6 \mathrm{C}}{ }^{+} \mathrm{CD} 115^{+}\right)$. (b) Frequencies of pDCs $\left(\mathrm{CD} 45^{+} \mathrm{SiglecH}{ }^{+} \mathrm{BST} 2^{+} \mathrm{CCR}{ }^{+}\right)$in the spleens, mesenteric lymph nodes (MLNs), and small intestine intraepithelial lymphocytes (IELs) from SPF and GF mice. (c) Frequencies of thymic pDCs $\left(\right.$ SiglecH $\left.{ }^{+} \mathrm{CCR}^{+}\right)$and total numbers of live thymocytes in SPF and GF mice. Error bars represent the mean \pm s.e.m. (d) Frequencies and numbers of pDCs $\left(\mathrm{Ly}_{49 \mathrm{Q}}{ }^{\text {hi SiglecH }}{ }^{+}\right.$) in the BM of SPF and GF mice. Other gates show immature pDCs and pDC progenitors (Ly49Q int and Ly $49 Q^{\text {lo }}$, respectively). (a-d) Data are combined from or are representative of two or three independent experiments with three to nine mice per experiment. $P$-values indicate statistical significance.

\section{Microbiota promotes trafficking of $\mathrm{CCR}^{\mathrm{hi}} \mathrm{pDCs}$ through CCL2}

It has been reported that $\mathrm{pDC}$ egress from $\mathrm{BM}$ in steady state depends on CCR5 and, in part, CCR2 ${ }^{6}$ In C57BL/6 mice, pDCs 
a

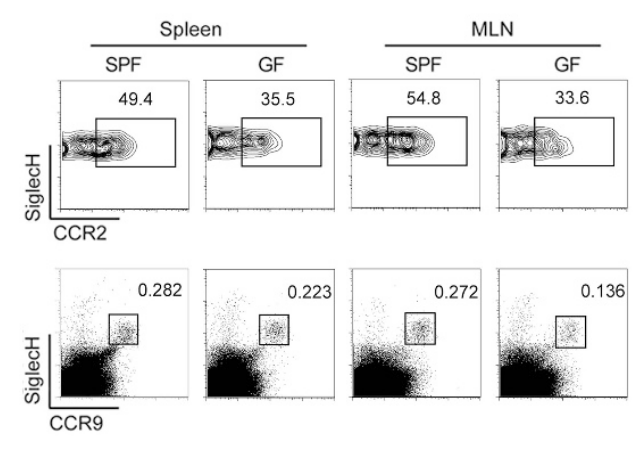

C

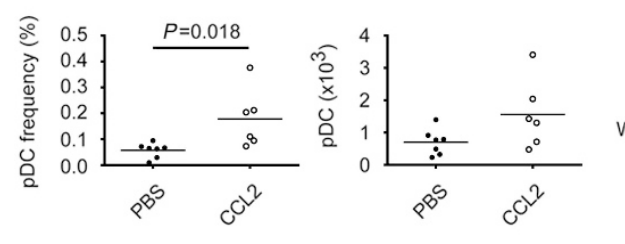

b
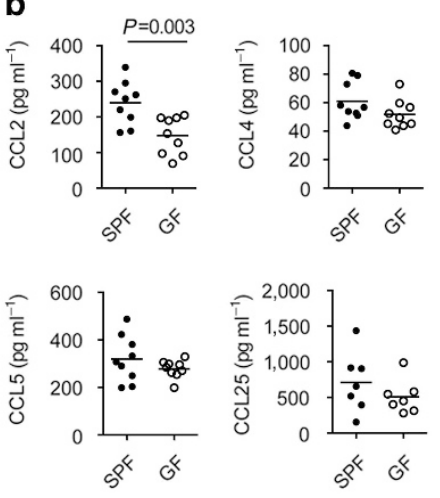

d

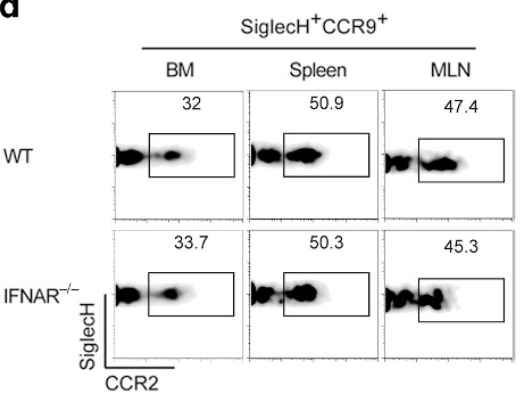

Figure 2 CCR2 ${ }^{\text {hi }}$ plasmacytoid dendritic cells ( $(\mathrm{PDCs})$ and systemic levels of CCL2 are reduced in germ-free (GF) mice. (a) CCR2 expression on pDCs $\left(\mathrm{SiglecH}^{+} \mathrm{CCR}^{+}\right.$) from the spleens and mesenteric lymph node (MLN) of specific pathogen-free (SPF) and GF mice. (b) CCL2, CCL4, CCL5, and CCL25 levels in sera from SPF and GF mice. (c) C57BL/6 mice were injected intraperitoneally with recombinant CCL2 or phosphate-buffered saline (PBS); pDC frequencies and numbers were measured in peritoneal exudates. (d) CCR2 expression on pDCs (SiglecH ${ }^{+} \mathrm{CCR}^{+}$) from the bone marrow (BM), spleen and MLN of wild-type (WT) and Ifnar ${ }^{-1-}$ mice. (a-d) Data are combined from or are representative of two or three independent experiments with two to nine mice per experiment. The $P$-value indicates statistical significance.

uniformly express CCR5, whereas, based on the amount of CCR2 expressed, pDCs can be divided into two distinct subsets, CCR $2^{\text {lo }}$ and CCR2 $2^{\text {hi }} 6$ Thus, we sought to investigate whether lack of microbiota impairs pDC trafficking by affecting CCR5 and/or CCR2 expression. GF mice had normal levels of CCR5 on pDCs from the BM, spleen, and MLN (data not shown). However, we noted that the ratios of CCR2 $2^{\text {lo }}$ and CCR $2^{\text {hi }}$ pDCs differed between SPF and GF mice, and that GF mice had a consistent reduction in CCR $2^{\text {hi }}$ pDCs in the periphery (Figure 2a). We further analysed the systemic levels of CCR5 ligands (CCL4 and CCL5) and the CCR2 ligand CCL2 in SPF and GF mice. We found that CCL2 but not CCL4 or CCL5 was lower in GF mice (Figure 2b), suggesting that microbiota may be essential for constitutive production of CCL2 that drives migration or accumulation of CCR $2^{\text {hi }}$ pDCs in peripheral compartments. To determine whether CCL2 promotes pDC trafficking, mice were injected intraperitoneally with recombinant CCL2 or phosphate-buffered saline (PBS) and $\mathrm{pDC}$ numbers in peritoneal exudates were measured. Results showed an increase in pDC frequency and number in peritoneal exudates, indicating that $\mathrm{pDCs}$ can be recruited by CCL2 (Figure 2c).

As microbiota has been shown to promote tonic IFN-I signaling in mononuclear phagocytes ${ }^{12,28,29}$ and IFN-I is required for pDC trafficking, ${ }^{30}$ we asked whether the reduction of CCR $2{ }^{\text {hi }}$ pDCs in GF mice was related to impaired IFN-I signaling in pDCs. However, WT and Ifnar ${ }^{-1-}$ mice had comparable frequencies of CCR $2^{\text {lo }}$ and CCR $2^{\text {hi }}$ pDCs in the BM, spleen and MLN (Figure 2d). The importance of CCR9 in pDC trafficking has also been documented; ${ }^{7,8}$ therefore, we evaluated CCR9 expression and systemic levels of CCL25 in SPF and GF mice. CCR9 expression was normal on pDCs from GF mice (Figure 2a) and serum CCL25 levels were comparable in SPF and GF mice (Figure 2b). Thus, these data suggested that the peripheral reduction of pDCs in GF mice, in particular the CCR $2{ }^{\text {hi }}$ subset, was due to lower levels of CCR2 ligands rather than defective expression of CCR5, CCR9, and their ligands or defective IFN-I signaling.

\section{CCR2 deficiency affects pDC trafficking in steady state}

To corroborate the role of CCR2 in pDC trafficking in steady state, we examined $\mathrm{Ccr}^{-/-}$mice. ${ }^{31}$ We found that $\mathrm{pDC}$ frequencies and numbers in the spleen and blood were reduced $\sim 50 \%$ in $\mathrm{Ccr}^{-/-}$mice (Figure 3a and b). To further demonstrate a role for CCR2 signaling in maintaining the CCR $2^{\text {hi }}$ subset in the periphery, we examined a recently generated line of CCR2-eGFP gene-targeted reporter mice. ${ }^{32}$ $C c r 2^{e G F P /+}$ mice harbored eGFP ${ }^{\text {lo }}$ and eGFP hi pDCs subsets, which corresponded to those observed with CCR2 staining (Figure 3c and data not shown). In most compartments of $C c r 2^{e G F P /+}$ mice examined, the ratio of eGFP ${ }^{\text {lo }}$ to $\mathrm{eGFP}^{\text {hi }}$ pDCs was 1:1, whereas in the BM there was a trend toward more $\mathrm{eGFP}^{\mathrm{lo}}$ than $\mathrm{eGFP}^{\text {hi }}$ pDCs (Figure 3c). However, more eGFP ${ }^{\text {hi }}$ $\mathrm{pDC}$ were present in the $\mathrm{BM}$ of $C \mathrm{cr} 2^{\text {eGFP/eGFP }}$ mice, which are 
a

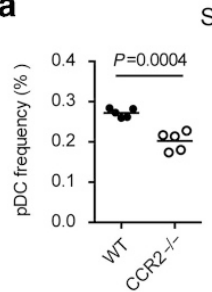

Spleen

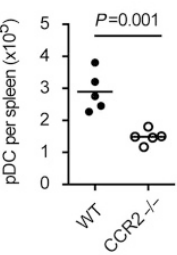

C
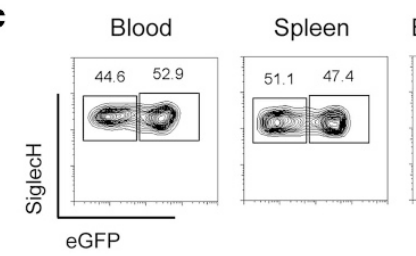

b

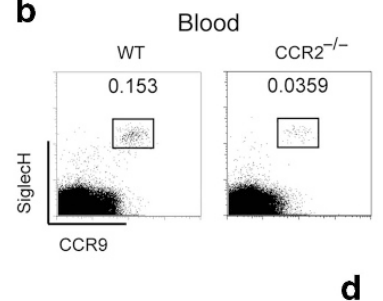

d Bone marrow
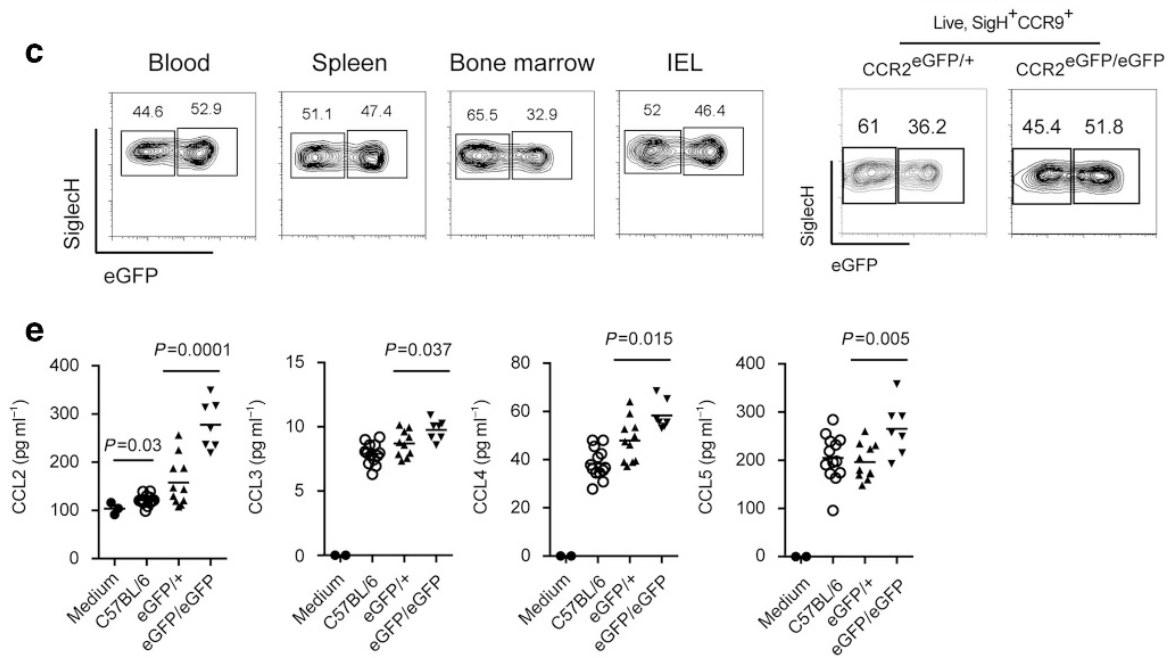

Figure 3 Plasmacytoid dendritic cell ( $\mathrm{pDC}$ ) homeostasis is altered in $\mathrm{Ccr}^{-/-}$mice. (a) pDC frequencies (SiglecH ${ }^{+} \mathrm{CCR}^{+}$) and numbers in the spleens of C57BL/6 (wild type, WT) and $\mathrm{Ccr}^{-/-}$mice. (b) Frequencies of $\mathrm{pDCs}\left(\right.$ SiglecH ${ }^{+} \mathrm{CCR}^{+}$) in peripheral blood from WT and Ccr2 ${ }^{-/-}$mice. (c) Blood, spleen, bone marrow (BM) and intraepithelial lymphocytes (IELs) were isolated from Ccr2 ${ }^{e G F P /+}$ mice and analyzed by flow cytometry. Graphs show two populations of $\mathrm{SiglecH}^{+} \mathrm{CCR}^{+}$pDCs based on enhanced green fluorescent protein (eGFP) expression in all compartments. (d) Frequencies of eGFPlo and eGFPhi pDCs in the BM of Ccr2 ${ }^{\text {eGFP/+ }}$ and Ccr2 ${ }^{\text {eGFPleGFP }}$ mice. (e) Systemic levels of CCL2, CCL3, CCL4 and CCL5 in WT, CCr2 ${ }^{\text {eGFP/+ }}$ and Ccr2 ${ }^{\text {eGFP/eGFP }}$ mice. Medium controls are included to indicate background. (a-d) Data are representative of two or three experiments with three to five mice per experiment. (e) Data are combined from two independent experiments $(n=7-13)$. $P$-values indicate statistical significance.

deficient for CCR2, such that eGFP ${ }^{\text {hi }}$ and $\mathrm{eGFP}^{\mathrm{lo}}$ pDCs were almost equally represented (Figure 3d); this implied that CCR2 signaling preferentially promotes homeostatic trafficking of CCR $2^{\text {hi }}$ pDCs.

CCL2 levels were also elevated in Ccr2 $2^{\text {eGFP/eGFP }}$ mice (Figure 3e), probably due to reduced usage and subsequent degradation. $\mathrm{Ccr} 2$ and $\mathrm{Ccr} 5$ genes are adjacent to each other on chromosome 9. As CCR5 has a major role in pDC egress from BM in steady state, ${ }^{6}$ we verified that CCR 5 expression levels on pDCs were normal in both $\mathrm{Ccr}^{-/-}$and $\mathrm{Crr} 2^{e G F / e G F P}$ mice (data not shown). Furthermore, as CCR5 ligands are made by various cell types, some of which may be altered or absent in the periphery of $\mathrm{Ccr}^{-/-}$mice, we investigated whether lack of CCR2 results in impaired production of CCR5 ligands. In fact, systemic levels of CCR5 ligands were higher in $\mathrm{Ccr} 2^{e G F P / e G F P}$ mice relative to $\mathrm{Ccr} 2^{e G F P /+}$ and WT mice (Figure 3e), suggesting that CCR2 deficiency does not impair CCR5 signaling. Altogether, these results indicate that CCR2 deficiency leads to a reduction in peripheral pDCs.

\section{Mononuclear phagocytes are required for the production of CCR2 ligands and PDC homeostasis}

CCL2 is produced by several cell types including mononuclear phagocytes and $\mathrm{T}$ cells, and is rapidly expressed by BM mesenchymal and colonic stromal cells in response to circulating bacterial infection, Toll-like receptor, and nucleotide oligomerization domain (NOD)-like receptor ligands. ${ }^{33,34}$ As mononuclear phagocytes can produce CCL2 and are primed by microbiota, ${ }^{16,28,29,33}$ we tested whether they had a role in the production of CCR2 ligands that drive $\mathrm{pDC}$ trafficking in steady state. We injected WT C57BL/6 mice with liposomes containing PBS or Clod, to eliminate mononuclear phagocytes. Two days after liposome injection, we observed a $\sim 50 \%$ reduction in total cellularity and pDC numbers in spleens of Clod-treated mice (Figure 4a). The CCR2 $2^{\text {hi }}$ pDC subset appeared to be most affected by Clod treatment (Figure 4b). Analyses of MLN and inguinal LN from PBS and Clod-treated mice also revealed a reduction in pDCs, in particular the CCR ${ }^{\text {hi }}$ subset (Figure 4c). In addition, we measured CCR2 and CCR5 ligands in serum from PBS and Clod-treated mice, and found that serum CCL2 was strongly reduced after Clod administration (Figure 4d), confirming the requirement of mononuclear phagocytes for homeostatic levels of CCL2.

Clod treatment also reduced serum CCL4 (Figure 4d). As we previously reported that pDC depletion has an impact on CCL4 levels, ${ }^{35}$ it is possible that lower levels of CCL4 in Clod-treated mice were a consequence of reduced numbers of pDCs. In contrast, serum CCL3 and CCL5 were increased in Clod-treated mice (Figure 4d) perhaps because of reduced consumption by pDCs and mononuclear phagocytes, which express CCR5. ${ }^{36}$ 
a
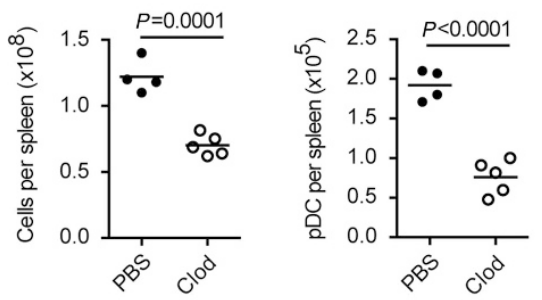

C
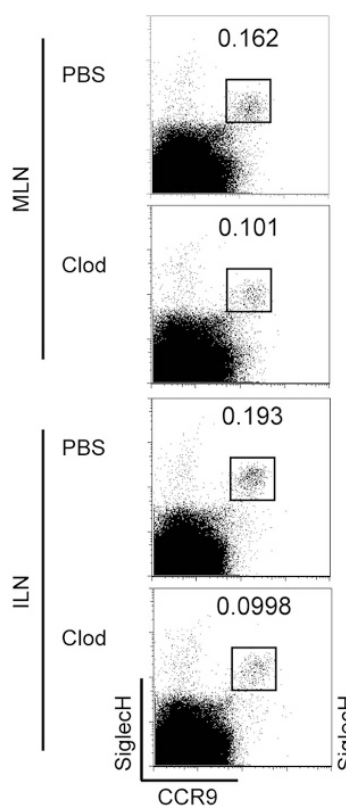

e

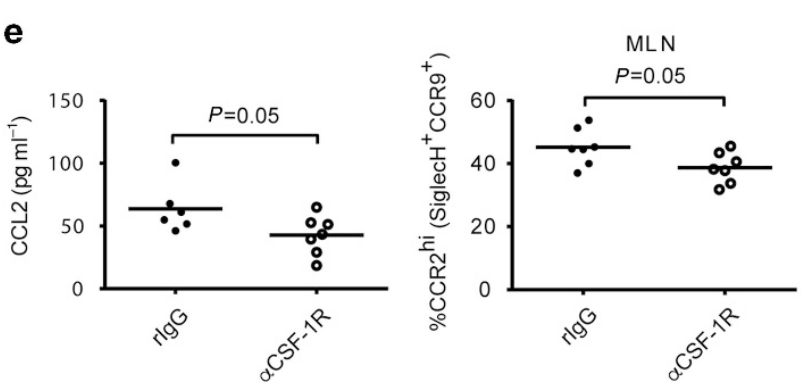

b

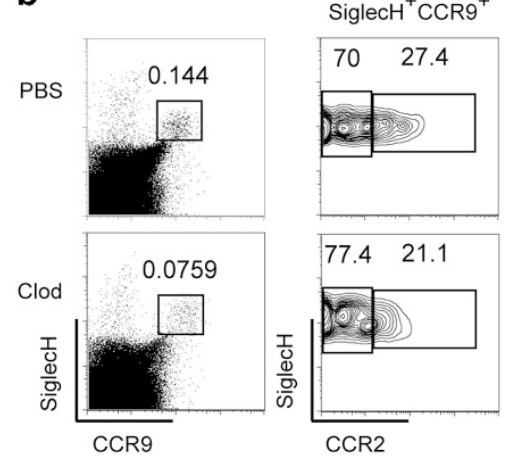

d
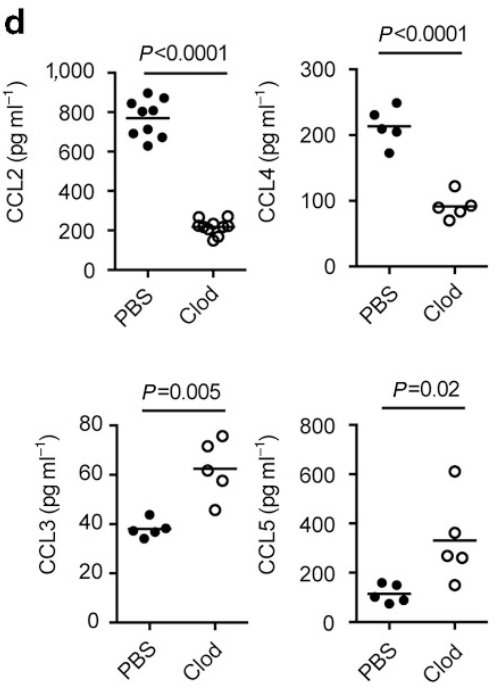

Figure 4 Mononuclear phagocytes produce CCR2 ligands and are required for plasmacytoid dendritic cell (pDC) homeostasis. (a-d) C57BL/6 mice were injected intravenously with phosphate-buffered saline (PBS) or clodronate (Clod) liposomes. Tissues and sera were analyzed $48 \mathrm{~h}$ post injection. (a) Total cellularity and numbers of pDCs $\left(\right.$ SiglecH $\left.{ }^{+} \mathrm{CCR} 9^{+}\right)$in spleens. (b) Plots show frequencies of pDCs and frequencies of CCR2 ${ }^{\text {lo }}$ and CCR $2^{\text {hi }}$ pDC subsets in spleens. (c) Plots show frequencies of pDCs, and CCR $2^{\text {lo }}$ and CCR2 ${ }^{\text {hi }}$ pDC subsets in mesenteric lymph node (MLN) and inguinal LN (ILN). (d) Systemic levels of CCL2, CCL3, CCL4 and CCL5. (e) C57BL/6 mice were injected intraperitoneally with anti-CSF-1R monoclonal antibody (mAb) or isotype control antibody (rlgG1) every 3 days for a total of seven doses. Mice were analyzed on day 21. Graphs show serum levels of CCL2 and frequencies of CCR2 $2^{\text {hi }}$ pDCs in MLN. (a-e) Data are representative of two independent experiments with two to nine mice per experiment. $P$-values indicate statistical significance.

Because of the toxicity associated with Clod, we also evaluated pDC numbers and serum CCL2 levels in C57BL/ 6 mice treated with anti-CSF-1R mAb, which reduces some mononuclear phagocyte subsets ${ }^{37}$. Serum CCL2 levels and CCR $2^{\text {hi }}$ pDCs in MLN were partially reduced (Figure 4e), although, in contrast to Clod treatment, anti-CSF-1R mAb did not significantly impact spleen or LN pDC numbers (data not shown). It is likely that the limited effect of anti-CSF-1R mAb treatment on CCL2 levels and pDC numbers is related to the incomplete depletion of monocytes and several tissue resident macrophage populations. ${ }^{37}$ Altogether, these data demonstrate that mononuclear phagocytes are required for the production of CCL2 that promotes pDC trafficking and distribution in steady state. We postulate that in steady state, CCL2 may be 


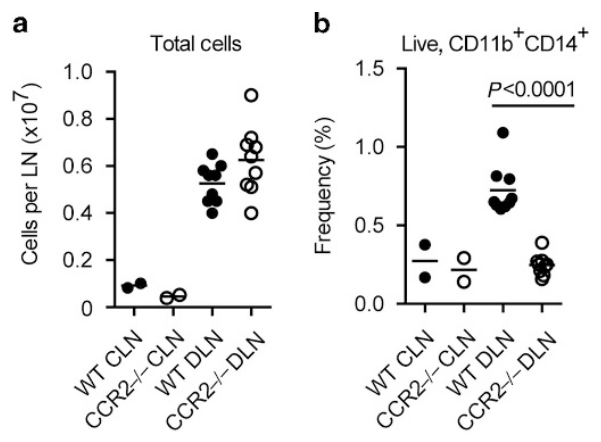

C

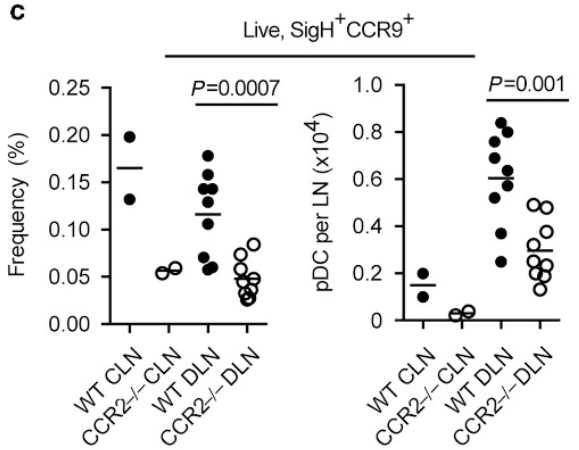

d
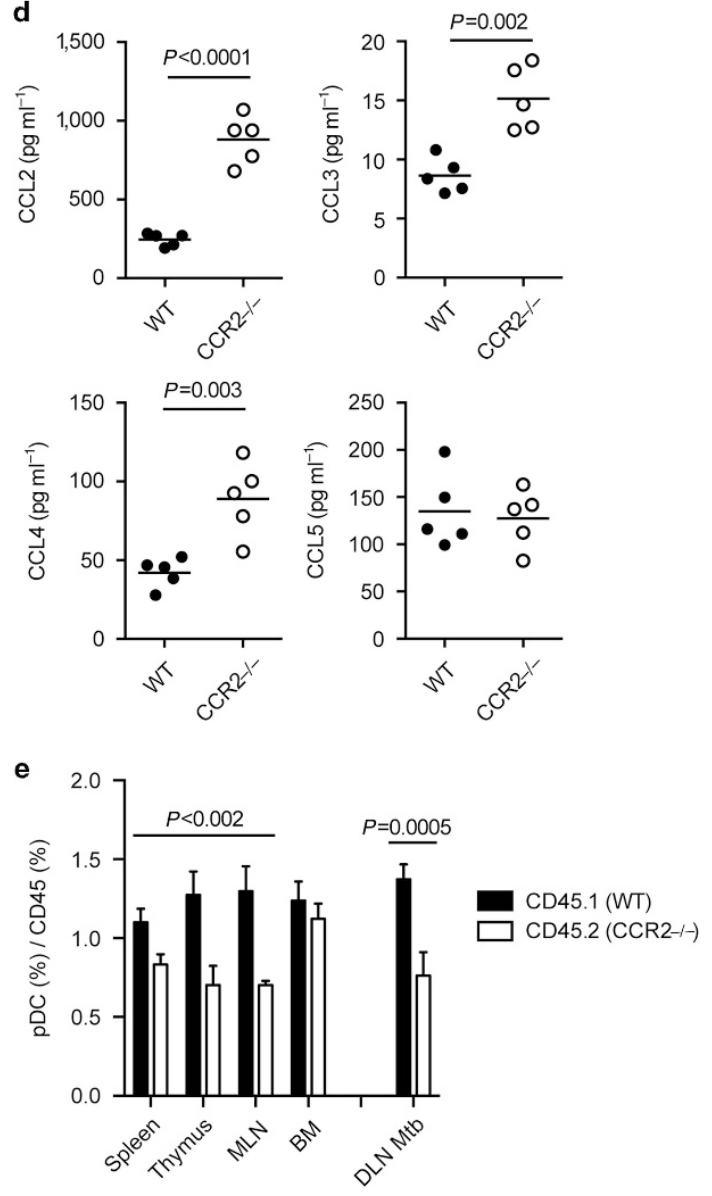

Figure 5 CCR2 deficiency impacts plasmacytoid dendritic cell (pDC) recruitment during inflammation. (a-d) Wild-type (WT) and $\mathrm{Ccr}^{-1}$ mice were injected in the footpad with heat-killed M. tuberculosis (Mtb). (a) Total cells in contralateral lymph node (CLN) and draining popliteal lymph nodes (DLNs). (b) Frequencies of myeloid cells $\left(\mathrm{CD} 11 \mathrm{~b}^{+} \mathrm{CD} 14^{+}\right)$in CLN and DLN. (c) Frequencies and numbers of pDCs $\left(\right.$ SiglecH $\left.{ }^{+} \mathrm{CCR}^{+}\right)$in $\mathrm{CLN}$ and DLN. (d) Systemic levels of CCL2, CCL3, CCL4 and CCL5 in WT and $\mathrm{Ccr}^{-1-}$ mice injected with Mtb. (e) Mixed bone marrow (BM) chimeras were analyzed 8 weeks after reconstitution for CD45.1 ${ }^{+}$(WT) and $\mathrm{CD} 45.2^{+}\left(\mathrm{Ccr}^{-/}\right)$pDCs $\left(\right.$SiglecH ${ }^{+}$Ly49Q $\left.{ }^{+}\right)$in the spleen, thymus, mesenteric lymph node (MLN) and BM or in DLN after Mtb injection. Graphs show the ratio of CD45.1 $1^{+}$or CD45.2 ${ }^{+}$pDCs (\%) to total CD $45.1^{+}$or CD45.2 ${ }^{+}$cells (\%). (a-c) Data are combined from two experiments with four to five mice per experiment. CLN were pooled from mice in each experiment (experiment one: $n=4$; experiment two: $n=5$ ). (d) Data are representative of two experiments with four to five mice per experiment. (e) Graphs show mean values \pm s.d. $(n=4)$. $P$-values indicate statistical significance.

largely produced by mononuclear phagocytes of the gut. Supporting this hypothesis, we did not detect CCR2 expression on intestinal intraepithelial pDCs using anti-CCR2 Ab (data not shown), whereas we could clearly see both $\mathrm{CCR} 22^{\mathrm{lo}}$ and CCR $2^{\text {hi }} \mathrm{pDCs}$ in intraepithelial lymphocytes from $\mathrm{Ccr} 2^{e G F P /+}$ mice (see Figure 3c). This result suggests that gut pDCs are exposed to a high concentration of CCL2, such that CCR2 is occupied or downregulated and therefore undetectable by cell surface staining.

\section{CCR2 deficiency impacts pDC trafficking during inflammation}

We next assessed the contribution of CCR2 in pDC recruitment under inflammatory conditions. We used a local inflammation model based on footpad injection with heat-killed Mycobacterium tuberculosis, which causes rapid recruitment of pDCs to draining popliteal LNs (DLN). ${ }^{38,39}$ On the day of analysis, absolute numbers of cells from DLN of $\mathrm{Ccr}^{-/-}$mice were comparable to WT mice (Figure 5a), but, as expected, $\mathrm{Ccr} 2^{-/-}$ mice had a major reduction in $\mathrm{CD}_{11 \mathrm{~b}}{ }^{+} \mathrm{CD} 14^{+}$cells (Figure 5b). pDC numbers were increased in DLN from both groups of mice relative to contralateral LN, but DLN from $\mathrm{Ccr}^{-/-}$mice contained 50\% less pDCs than DLN from WT mice (Figure 5c), suggesting the importance of CCR2 in pDC recruitment during inflammation.

pDC migration during inflammation is controlled by multiple chemokines, such as CCR5 ligands, ${ }^{5}$ which are made by a variety of cell types. Thus, it was possible that some of these cells may be altered or absent in the periphery of $\mathrm{Crr} 2^{-/-}$mice, and that the pDC migration defect was secondary to a defect of another cell type or another chemokine. To exclude this possibility, we measured serum levels of CCR5 ligands in WT and $\mathrm{Crr} 2^{-/-}$mice after M. tuberculosis injection. CCL5, CCL4, CCL3 and CCL2 levels in $\mathrm{Ccr}^{-/-}$mice were similar to or higher relative to WT mice (Figure 5d). To further address whether the pDC deficit in $\mathrm{Crr} 2^{-/-}$mice was intrinsic or extrinsic, we generated WT and $\mathrm{Crr}^{-/-}$mixed BM chimeras using C57BL/6 mice as recipients. Mice were analysed 8 weeks after reconstitution under steady state and inflammatory 
a

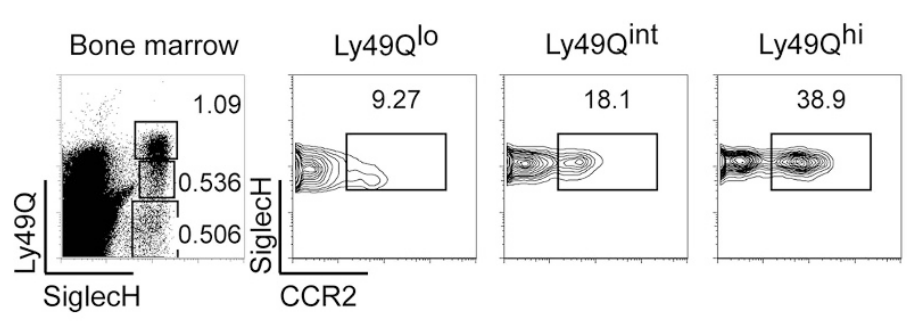

b
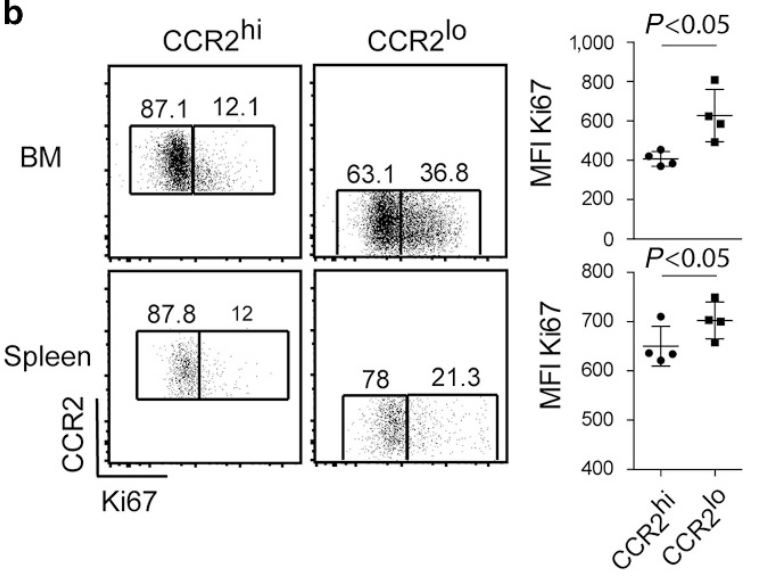

e

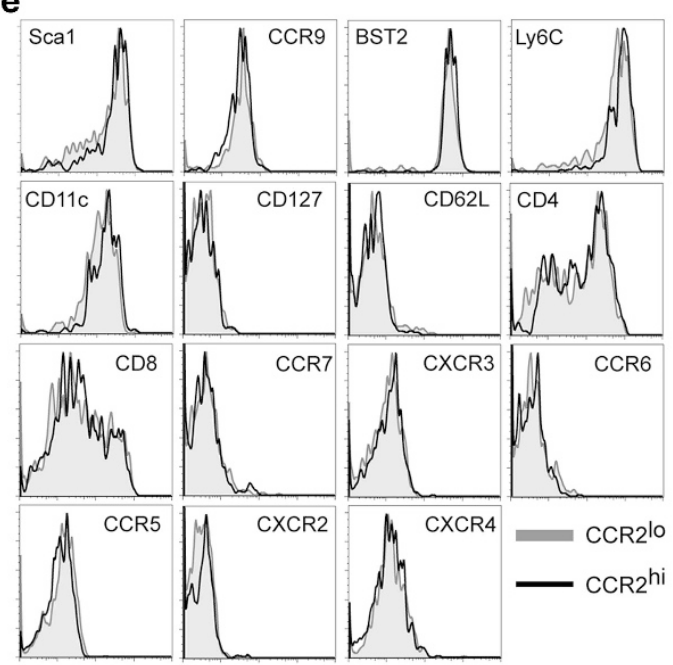

Figure $6 \mathrm{CCR} 2^{10}$ and CCR2 ${ }^{\text {hi }}$ plasmacytoid dendritic cells (pDCs) are functionally distinct. (a) Bone marrow (BM) from C57BL/6 mice was stained for Ly49Q, SiglecH and CCR2. Graphs show percentages of CCR2 ${ }^{\text {hi }}$ subsets in mature pDCs (Ly49Q ${ }^{\text {hi }}$ iglecH $\left.^{+}\right)$, immature pDCs $\left({\text {Ly } 49 Q^{\text {int }} \text { SiglecH }}^{+}\right)$, and progenitors $\left(\mathrm{Ly}_{49 \mathrm{Q}}{ }^{\mathrm{lo}} \mathrm{SiglecH}{ }^{+}\right.$). (b) BM and spleen cells were collected and stained for Ki67 to measure proliferative capacity. Dot plots show CCR2 ${ }^{\text {hi }}$ and $\mathrm{CCR}^{\mathrm{lo}} \mathrm{pDCs}\left(\mathrm{SiglecH}^{+} \mathrm{B} 22 \mathrm{O}^{\mathrm{hi}}\right.$ ), and graphs show mean fluorescence intensities (MFIs) of Ki67 expression. (c) CCR2 ${ }^{\mathrm{lo}}$ and CCR2 ${ }^{\mathrm{hi}} \mathrm{pDCs}^{\mathrm{C}}$ $\left(\mathrm{CCR}^{+}{ }^{+} \mathrm{SiglecH}^{+} \mathrm{B} 22 \mathrm{O}^{\mathrm{hi}}\right)$ were sorted from BM of $\mathrm{CCr} 2^{\mathrm{eGFP} /+}$ mice and incubated with Toll-like receptor (TLR) agonists overnight. Supernatants were collected and analyzed for interferon (IFN)- $\alpha$ and interleukin (IL)-6. Error bars represent the mean \pm s.e.m. (d) CCR2 ${ }^{\text {hi }}$ and CCR2 ${ }^{\text {lo }}$ pDCs

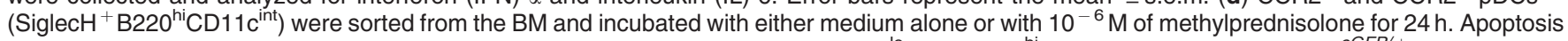
was measured by Annexin-V and 7AAD staining. (e) Phenotypic analysis of CCR2 $2^{\mathrm{lo}}$ and CCR2 $2^{\mathrm{hi}} \mathrm{pDCs}$ from spleens of $C c r 2^{\mathrm{eGFP} /+}$ mice.

(a-e) Data are representative of two or three experiments with at least three to five mice per experiment. $P$-values indicate statistical significance.

conditions. In naive mice, $\mathrm{Ccr}^{-/-}$pDCs were reduced in the spleen, thymus, and MLN compared with WT pDCs but ratios of WT and $\mathrm{Ccr}^{-/-}$pDCs were similar in BM (Figure 5e). After M. tuberculosis injection, DLN contained fewer $\mathrm{Ccr}^{-/-}$pDCs (Figure 5e). Taken together, these data suggest that CCR2 deficiency affects pDC homing in steady state and during inflammation in a cell-intrinsic manner.

\section{$\mathrm{CCR}^{\mathrm{hi}}$ and $\mathrm{CCR}^{\mathrm{lo}} \mathrm{pDC}$ s are functionally different}

Finally, we sought to determine whether CCR2 ${ }^{\text {hi }}$ and CCR $2^{\text {lo }}$ pDCs that are differentially responsive to microbiota-induced CCL2 are phenotypically and/or functionally different. As pDCs develop in the BM through sequential maturation steps ${ }^{40}$ we examined whether CCR2 levels correlate with pDC maturation in BM using markers such as SiglecH, CCR9, and Ly49Q (Figure 6a and data not shown). The majority of
pDC precursors were CCR $2^{\text {lo }}$ or eGFP $^{\text {lo }}(80-90 \%)$; in comparison, $50-60 \%$ of mature pDCs were CCR $2^{\text {lo }}$ or eGFP ${ }^{\text {lo }}$ (Figure 6a and data not shown). Moreover, Ki67, which is a marker of proliferative capacity, was less expressed in CCR $2{ }^{\text {hi }}$ $\mathrm{pDCs}$ compared to CCR $2^{\mathrm{lo}} \mathrm{pDCs}$ in BM (Figure $6 \mathbf{b}$ ). Thus, $\mathrm{CCR} 22^{\mathrm{hi}} \mathrm{pDCs}$ have a more mature phenotype than CCR $2^{\text {lo }}$ pDCs.

We next evaluated the ability of BM CCR $2^{\text {lo }}$ and CCR $2^{\text {hi }}$ pDCs to respond to Toll-like receptor-7/9 stimulation ex vivo. pDC subsets were sorted from BM of CCR2 ${ }^{\mathrm{eGFP} /+}$ mice and cultured overnight with $\mathrm{CpGA}, \mathrm{CpGB}$, Imiquimod or murine cytomegalovirus. CCR $2^{\mathrm{lo}}$ pDCs produced more IFN- $\alpha$ than CCR2 ${ }^{\text {hi }}$ pDCs after stimulation with CpGA and murine cytomegalovirus, a feature of immature pDCs. In contrast, CCR $2^{\text {hi }}$ pDCs produced more proinflammatory cytokines such as interleukin- 6 in response to Imiquimod and $\mathrm{CpGB}$, a feature 
of mature pDCs (Figure 6c). We also tested the susceptibility of $\mathrm{BM}$ CCR $2^{\text {lo }}$ and CCR $2^{\text {hi }}$ pDCs to spontaneous and glucocorticoid-induced apoptosis, and found that CCR2 $2^{\text {hi }}$ cells were more susceptible to both (Figure 6d). We finally asked whether CCR2 ${ }^{\mathrm{lo}}$ and $\mathrm{CCR} 2^{\mathrm{hi}}$ pDCs differed in the periphery. Splenic CCR $2^{\text {lo }}$ and CCR2 ${ }^{\text {hi }}$ pDCs expressed similar levels of the markers: Sca1, CCR9, BST2, Ly6C, CD11c, CD127, CD62L, CD4, CD8, CCR7, CXCR3, CCR6, CCR5, CXCR2 and CXCR4 (Figure 6e). However, CCR $2^{\text {hi }}$ pDCs expressed less Ki67 and were more prone to apoptosis (Figure $\mathbf{6 b}$ and data not shown). Altogether, these results suggest that CCR $2{ }^{\text {hi }} \mathrm{pDCs}$ are functionally different from CCR $2^{\mathrm{lo}} \mathrm{pDC}$, are more capable of producing proinflammatory cytokines, and are prone to apoptosis.

\section{DISCUSSION}

Our study establishes a role for the microbiota in inducing homeostatic serum levels of CCL2 that control trafficking and peripheral distribution of $\mathrm{pDCs}$ in the steady state. Although CCR2 is expressed on several immune cell types, our results indicate that CCL2 acts directly on pDCs. Homeostatic CCL2 appears to have a prominent impact on the CCR2 $2^{\text {hi }}$ subset of pDCs, which are more capable of producing proinflammatory cytokines and more prone to apoptosis than the CCR $2^{\mathrm{lo}}$ subset. Moreover, our study also demonstrates that CCR2 is an important chemokine receptor for controlling $\mathrm{pDC}$ migration during inflammation induced by products of pathogenic bacteria.

Colonization of the intestine with commensal microbes is essential for normal development and homeostasis of the cellular and humoral immune system. ${ }^{11-18}$ Several studies have shown that the gut microbiota has an impact on antigen presenting cells and mononuclear phagocytes. ${ }^{29,41}$ Similarly, intestinal Bacteroides fragilis, segmented filamentous bacteria, and Clostridia drive differentiation of Th1, Th17, and regulatory T cells, respectively. ${ }^{42-44}$ Moreover, the gut microbiota is a major driving force for differentiation of mucosal plasma cells and IgA production. ${ }^{21,45}$ Microbiota has also been shown to induce intestinal epithelial cells to secrete chemokines, such as CXCR2 ligands, which attract mast cells into the intestine. ${ }^{46}$ Recently, commensal-derived signals were shown to provide tonic stimulation that establishes the activation threshold of the innate immune system. ${ }^{28,29}$ Moreover, commensal microbial products, such as polysaccharide A, were found to endow pDCs with the capacity to induce interleukin-10 producing regulatory $\mathrm{T}$ cells. ${ }^{20}$ Our study reveals a novel function of the microbiota in the induction of constitutive levels of CCL2, which promote the circulation and distribution of pDCs and CCR2 ${ }^{+}$inflammatory monocytes. Although CCL2 has been shown to be a key chemokine in the recruitment of CCR $2{ }^{+}$inflammatory monocytes, which are involved in the pathogenesis of multiple diseases, ${ }^{25,47,48}$ this is the first time that CCL2 is shown to be important in the steadystate circulation of pDCs. It will be important to determine whether CCL2 is induced by selective commensals. It is also noteworthy that lack of microbiota did not result in a general reduction of serum chemokines, as serum levels of CCL4, CCL5, and CCL25 were similar in GF and SPF mice. Thus, CCL2 is a distinctive product of host immune systemmicroflora interaction.

We envision that microbiota activates cells with a prominent capacity to secrete CCL2, in particular mononuclear phagocytes, although we do not exclude other cells, especially in the intestine, such as colonic stromal cells, which were found to produce CCL2 in response to pathogenic bacteria in a Nod2dependent manner. ${ }^{34}$ Supporting the role of intestinal mononuclear phagocytes in CCL2 production in response to microbiota priming, we did not detect CCR2 expression on gut pDCs using anti-CCR2 Ab (data not shown), whereas CCR2 mRNA was expressed as demonstrated by enhanced green fluorescent protein (eGFP) expression in gut pDCs from $\mathrm{Ccr} 2^{e G F P /+}$ mice. This discrepancy between mRNA and protein expression may indicate high concentrations of CCL2 in the gut, which downregulates CCR2, rendering it undetectable by cell surface staining. It is likely that mononuclear phagocytes are also necessary for CCL2 production during inflammation, acting directly at the site of inflammation. Here, CCL2 released by inflammatory monocytes may activate pDCs reaching the inflammatory site and CCR2 ${ }^{+}$ endothelial cells, promoting pDC adhesion to the endothelium and transendothelial migration. A similar mechanism has been shown for monocyte-mediated recruitment of neutrophils during arthritis. ${ }^{49}$

It was previously shown that pDCs include CCR $2^{\text {hi }}$ and CCR $2{ }^{\text {lo }}$ subsets, and that a partial defect of CCR2 expression secondary to Runx 2 deficiency contributes to retention of pDCs in the BM. ${ }^{6}$ Our study advances these observations, demonstrating that CCL2 controlling steady-state pDC circulation is dependent on microbiota and preferentially drives the trafficking of CCR 2 hi $\mathrm{pDCs}$. Moreover, we define functional differences between CCR $2^{\text {hi }}$ and CCR $2^{\text {lo }}$ subsets. CCR $2^{\text {hi }}$ pDCs produce more proinflammatory cytokines and are prone to spontaneous and glucocorticoid-induced apoptosis, whereas CCR $2{ }^{\mathrm{lo}} \mathrm{pDCs}$ produce more IFN-I and express Ki67. Thus, it appears that expression level of CCR2 somewhat correlates with pDC maturation, although it remains unclear whether CCR $2^{\text {lo }}$ and CCR2 ${ }^{\text {hi }}$ cells develop through sequential maturation steps or are distinct subsets.

In conclusion, our findings identify a novel role of microbiota in shaping the immune system, which consists of the induction of tonic levels of serum CCL2 that control trafficking and peripheral localization of pDCs.

\section{METHODS}

Mice and treatments. All animal studies were approved by the Washington University Animal Studies Committee. SPF male and female C57BL/6 mice (CD45.2) were bred in-house. Female C57BL/6 GF mice (7-9 weeks old) were bred in-house by the J. Gordon lab (Washington University, St. Louis, MO). Male and female Ccr $2^{-/-}$mice (CD45.2) were either purchased from the Jackson Laboratory or bred at Washington University School of Medicine (St. Louis, MO). Male C57BL/6 (CD45.1) mice were purchased from the Jackson Laboratory (Bar Harbor, ME) for mixed BM chimera experiments. Ifnar ${ }^{-1-}$ mice 
were kindly provided by A. French (Washington University School of Medicine). CCR2-eGFP reporter mice were generated and bred at Washington University School of Medicine. ${ }^{32}$ Liposomes containing PBS or Clod were generous gifts from the G. Randolph lab (Washington University School of Medicine). Liposomes were injected intravenously $48 \mathrm{~h}$ before analysis. Anti-CSF-1R mAb (M279) was kindly provided by Dr. Wenjun Ouyang (Amgen, San Francisco, CA). C57BL/6 WT mice were injected intraperitoneally with $400 \mu \mathrm{g}$ antiCSF-1R mAb or isotype control antibody (rIgG1) every 3 days for a total of 7 doses. C57BL/6 mice were injected with $300 \mathrm{ng}$ of recombinant CCL2 (R\&D Systems, Minneapolis, MN) or PBS intraperitoneally every other day for a total of three doses. Mice were analyzed $14 \mathrm{~h}$ after the final injection.

BM chimeras. BM from 6-week-old $\mathrm{Ccr}^{-/-}$mice (CD45.2) and C57BL/6 mice (CD45.1) was collected from the tibias and femurs. After red blood cell lysis, cells were counted and mixed at a 1:1 ratio and then injected into lethally irradiated (1,000 rad) C57BL/6 mice (CD45.2). Cells $\left(3 \times 10^{6}\right)$ of each genotype were injected intravenously $8 \mathrm{~h}$ after irradiation. BM chimeras were analyzed 8 weeks after reconstitution.

Inflammation assay. C57BL/6 mice, $\mathrm{Ccr} 2^{-/-}$mice, and mixed BM chimeras were injected in the hind footpads with $500 \mu \mathrm{g}$ of heat-killed M. tuberculosis (Difco, Franklin Lakes, NJ) on days -2 and 0. Serum, contralateral LN, and DLN were collected on day 1 for analysis.

Cell preparations. Cell suspensions of the spleen, thymus, and LN were prepared by collagenase $\mathrm{D}$ digestion and passage through nylon mesh cell strainers (BD Biosciences, San Jose, CA). The BM was collected from the tibias and femurs. Whole blood was isolated by cardiac puncture for serum and flow cytometry. Red blood cells were lysed with red blood cell lysis buffer (Sigma-Aldrich, St. Louis, MO). Intraepithelial cells from small intestine were isolated as described with modifications. ${ }^{50}$ Briefly, small intestines were sectioned after removal of Peyer's patches and incubated for $20 \mathrm{~min}$ at room temperature in Hank's balanced salt solution, $10 \%$ bovine calf serum, EDTA, and HEPES with mild agitation (two rounds). Supernatants were passed through nylon mesh strainers and centrifuged over a $44-67 \%$ Percoll gradient.

Antibodies, flow cytometry, and sorting. The following reagents were from BD Biosciences, eBioscience (San Diego, CA), or Biolegend (San Diego, CA): fluorochrome-labeled anti-CD45.1 (A20), antiCD45.2 (104), anti-CCR9 (eBioCW-1.2), anti-CD11c (HL3), antiSiglecH (551 or 440c), anti-B220 (RA3-6B2), anti-CD4 (GK1.5), antiCD8 $\alpha$ (53-6.7), anti-CD11b (M1/70), anti-Ly6C (AL-21), anti-CD14 (Sa2-8), anti-CD62L (MEL-14), anti-CCR7 (4B12), anti-CCR6 (140706), anti-CCR5 (HM-CCR5), anti-CXCR4 (2B11/CXCR4), antiCXCR2 (TG11/CXCR2), anti-CXCR3 (CXCR3-173), anti-BST2 (927), anti-CD127 (A7R34), and anti-Scal (D7). Anti-Ly49Q (2E6) and antiCCR2 (475301) were purchased from MBL (Woburn, MA) or R\&D Systems, respectively. Fc receptors were blocked before surface staining and dead cells were excluded with propidium iodide or by scatter. Staining for chemokine receptors, with the exception of CCR9, was achieved by incubating cells in complete medium containing $\mathrm{Fc}$ block and fluorochrome-conjugated antibodies for $30 \mathrm{~min}$ at $37^{\circ} \mathrm{C}$. Flow cytometry data were analyzed with FlowJo software (Tree Star, Ashland, OR). CCR $2^{\text {hi }}$ and CCR $2^{\text {lo }}$ pDCs from the BM and spleens of WT or CCR2 $2^{\mathrm{eGFP} /+}$ mice were sorted on a FACSAria (BD Biosciences).

Cell culture and stimulations. Primary cells were cultured in complete RPMI 1640 with $10 \%$ fetal calf serum, 1\% glutamax, $1 \%$ non-essential amino acids, $1 \%$ sodium pyruvate, and $1 \%$ kanamycin sulfate (GibcoInvitrogen, Carlsbad, CA). Sorted pDCs were stimulated overnight with CpGA $2216\left(6 \mu \mathrm{g} \mathrm{ml}^{-1}\right.$, InvivoGen, San Diego, CA), murine cytomegalovirus (multiplicity of infection 10:1), CpGB 1826 $\left(6 \mu \mathrm{g} \mathrm{ml}^{-1}\right.$, InvivoGen), or Imiquimod $(6 \mu \mathrm{M}$, InvivoGen). For apoptosis studies, BM and spleen pDCs were incubated with medium alone or with $10^{-6} \mathrm{M}$ methylprednisolone for $24 \mathrm{~h}$. Apoptosis was assessed by Annexin-V and 7-aminoactinomycin D staining. Proliferative capacity was measured by Ki67 staining.

Enzyme-linked immunosorbent assay and cytometric bead array. Serum samples from mice were collected and stored at $-20^{\circ} \mathrm{C}$ until analysis. Interleukin-6, CCL2, CCL3, CCL4, and CCL5 were measured by cytometric bead array (BD Biosciences). CCL25 and IFN- $\alpha$ levels were measured by enzyme-linked immunosorbent assay (SigmaAldrich and PBL Assay Science, Piscataway, NJ).

Statistical analysis. The statistical significance of differences in mean values was analyzed with unpaired, two-tailed Student's $t$-test. $P$-values $<0.05$ were considered statistically significant.

\section{ACKNOWLEDGMENTS}

We thank Y. Wang, S. Rowland, A. French, T. Geurs, E. Unanue, B. Calderon, D. Kreisel, H. Hartzler, E.J. Pearce, W. Lam, G. Randolph, E. Gautier and S. Ivanov (Washington University) for mice, reagents, injections, and thoughtful discussions; J.I. Gordon, P. Ahern, D. O'Donnell and M. Karlsson (Center for Genome Sciences \& Systems Biology, Washington University) for help with the experiments using GF mice; and $\mathrm{E}$. Lantelme and D. Brinja for cell sorting. M.S. was supported by K01DK095972 from NIDDK and H.L.M. is supported by F30 DK 112508. Research reported in this publication was also funded by the National Institute of Arthritis and Musculoskeletal and Skin Diseases, part of the National Institutes of Health, under Award Number P30AR048335.

\section{AUTHOR CONTRIBUTIONS}

M.S., H.L.M., R.S.-C. performed experiments. S.G. established mice lines and colonies. M. Cella provided critical ideas. M.S. and M. Colonna wrote the manuscript. M. Colonna supervised research.

\section{DISCLOSURE}

M.S. is an employee of Janssen Research \& Development LLC. All other authors declare no conflicts of interest.

c 2017 Society for Mucosal Immunology

\section{REFERENCES}

1. Iwasaki, A. \& Medzhitov, R. Control of adaptive immunity by the innate immune system. Nat. Immunol. 16, 343-353 (2015).

2. Swiecki, M. \& Colonna, M. The multifaceted biology of plasmacytoid dendritic cells. Nat. Rev. Immunol. 15, 471-485 (2015).

3. Reizis, B., Bunin, A., Ghosh, H.S., Lewis, K.L. \& Sisirak, V. Plasmacytoid dendritic cells: recent progress and open questions. Ann. Rev. Immunol. 29, 163-183 (2011).

4. Ganguly, D., Haak, S., Sisirak, V. \& Reizis, B. The role of dendritic cells in autoimmunity. Nat. Rev. Immunol. 13, 566-577 (2013).

5. Sozzani, S., Vermi, W., Del Prete, A. \& Facchetti, F. Trafficking properties of plasmacytoid dendritic cells in health and disease. Trends Immunol. 31, 270-277 (2010)

6. Sawai, C.M. et al. Transcription factor Runx2 controls the development and migration of plasmacytoid dendritic cells. J. Exp. Med. 210, 2151-2159 (2013).

7. Hadeiba, H. et al. Plasmacytoid dendritic cells transport peripheral antigens to the thymus to promote central tolerance. Immunity 36, 438-450 (2012)

8. Wendland, M. et al. CCR9 is a homing receptor for plasmacytoid dendritic cells to the small intestine. Proc. Natl Acad. Sci. USA 104, 6347-6352 (2007).

9. Clahsen, T., Pabst, O., Tenbrock, K., Schippers, A. \& Wagner, N. Localisation of dendritic cells in the gut epithelium requires MAdCAM-1. Clin. Immunol. 156, 74-84 (2014).

10. Sisirak, V. et al. CCR6/CCR10-mediated plasmacytoid dendritic cell recruitment to inflamed epithelia after instruction in lymphoid tissues. Blood 118, 5130-5140 (2011). 
11. Rescigno, M. Intestinal microbiota and its effects on the immune system. Cell. Microbiol. 16, 1004-1013 (2014).

12. Cho, H. \& Kelsall, B.L. The role of type I interferons in intestinal infection, homeostasis, and inflammation. Immunol. Rev. 260, 145-167 (2014).

13. Littman, D.R. \& Pamer, E.G. Role of the commensal microbiota in normal and pathogenic host immune responses. Cell Host Microbe 10, 311-323 (2011).

14. Kamada, N., Seo, S.U., Chen, G.Y. \& Nunez, G. Role of the gut microbiota in immunity and inflammatory disease. Nat. Rev. Immunol. 13, 321-335 (2013).

15. Hooper, L.V., Littman, D.R. \& Macpherson, A.J. Interactions between the microbiota and the immune system. Science 336, 1268-1273 (2012).

16. Bain, C.C. et al. Constant replenishment from circulating monocytes maintains the macrophage pool in the intestine of adult mice. Nat. Immunol. 15, 929-937 (2014).

17. Balmer, M.L. et al. Microbiota-derived compounds drive steady-state granulopoiesis via MyD88/TICAM signaling. J. Immunol. 193, 5273-5283 (2014).

18. Niess, J.H. \& Adler, G. Enteric flora expands gut lamina propria CX3CR1 + dendritic cells supporting inflammatory immune responses under normal and inflammatory conditions. J. Immunol. 184, 2026-2037 (2010).

19. Fujiwara, D. et al. Systemic control of plasmacytoid dendritic cells by CD8 + T cells and commensal microbiota. J. Immunol. 180, 5843-5852 (2008).

20. Dasgupta, S., Erturk-Hasdemir, D., Ochoa-Reparaz, J., Reinecker, H.C. \& Kasper, D.L. Plasmacytoid dendritic cells mediate anti-inflammatory responses to a gut commensal molecule via both innate and adaptive mechanisms. Cell Host Microbe 15, 413-423 (2014).

21. Tezuka, H. et al. Prominent role for plasmacytoid dendritic cells in mucosal T cell-independent IgA induction. Immunity 34, 247-257 (2011).

22. Deal, E.M., Lahl, K., Narvaez, C.F., Butcher, E.C. \& Greenberg, H.B. Plasmacytoid dendritic cells promote rotavirus-induced human and murine B cell responses. J. Clin. Invest. 123, 2464-2474 (2013).

23. Goubier, A. et al. Plasmacytoid dendritic cells mediate oral tolerance. Immunity 29, 464-475 (2008).

24. Li, H.S. et al. Cell-intrinsic role for IFN-alpha-STAT1 signals in regulating murine Peyer patch plasmacytoid dendritic cells and conditioning an inflammatory response. Blood 118, 3879-3889 (2011).

25. Auffray, C., Sieweke, M.H. \& Geissmann, F. Blood monocytes: development, heterogeneity, and relationship with dendritic cells. Ann. Rev. Immunol. 27, 669-692 (2009).

26. Serbina, N.V. \& Pamer, E.G. Monocyte emigration from bone marrow during bacterial infection requires signals mediated by chemokine receptor CCR2. Nat. Immunol. 7, 311-317 (2006).

27. Mildner, A., Yona, S. \& Jung, S. A close encounter of the third kind: monocyte-derived cells. Adv. Immunol. 120, 69-103 (2013).

28. Abt, M.C. et al. Commensal bacteria calibrate the activation threshold of innate antiviral immunity. Immunity 37, 158-170 (2012).

29. Ganal, S.C. et al. Priming of natural killer cells by nonmucosal mononuclear phagocytes requires instructive signals from commensal microbiota. Immunity 37, 171-186 (2012).

30. Asselin-Paturel, C. et al. Type I interferon dependence of plasmacytoid dendritic cell activation and migration. J. Exp. Med. 201, 1157-1167 (2005).
31. Boring, L. et al. Impaired monocyte migration and reduced type 1 (Th1) cytokine responses in C-C chemokine receptor 2 knockout mice. J. Clin. Invest. 100, 2552-2561 (1997).

32. Epelman, S. et al. Embryonic and adult-derived resident cardiac macrophages are maintained through distinct mechanisms at steady state and during inflammation. Immunity 40, 91-104 (2014).

33. Shi, C. et al. Bone marrow mesenchymal stem and progenitor cells induce monocyte emigration in response to circulating toll-like receptor ligands. Immunity 34, 590-601 (2011).

34. Kim, Y.G. et al. The Nod2 sensor promotes intestinal pathogen eradication via the chemokine CCL2-dependent recruitment of inflammatory monocytes. Immunity 34, 769-780 (2011).

35. Swiecki, M., Gilfillan, S., Vermi, W., Wang, Y. \& Colonna, M. Plasmacytoid dendritic cell ablation impacts early interferon responses and antiviral NK and CD8(+) T cell accrual. Immunity 33, 955-966 (2010).

36. Gautier, E.L. et al. Gene-expression profiles and transcriptional regulatory pathways that underlie the identity and diversity of mouse tissue macrophages. Nat. Immunol. 13, 1118-1128 (2012).

37. Hume, D.A. \& MacDonald, K.P. Therapeutic applications of macrophage colony-stimulating factor-1 (CSF-1) and antagonists of CSF-1 receptor (CSF-1R) signaling. Blood 119, 1810-1820 (2012).

38. Blasius, A., Vermi, W., Krug, A., Facchetti, F., Cella, M. \& Colonna, M. A cell-surface molecule selectively expressed on murine natural interferonproducing cells that blocks secretion of interferon-alpha. Blood 103, 4201-4206 (2004).

39. Srivatsan, S., Swiecki, M., Otero, K., Cella, M. \& Shaw, A.S. CD2associated protein regulates plasmacytoid dendritic cell migration, but is dispensable for their development and cytokine production. J. Immunol. 191, 5933-5940 (2013).

40. Reizis, B. Regulation of plasmacytoid dendritic cell development. Curr. Op. Immunol. 22, 206-211 (2010).

41. Diehl, G.E. et al. Microbiota restricts trafficking of bacteria to mesenteric lymph nodes by CX(3)CR1(hi) cells. Nature 494, 116-120 (2013).

42. Mazmanian, S.K., Liu, C.H., Tzianabos, A.O. \& Kasper, D.L. An immunomodulatory molecule of symbiotic bacteria directs maturation of the host immune system. Cell 122, 107-118 (2005).

43. Ivanov, I.I. et al. Induction of intestinal Th17 cells by segmented filamentous bacteria. Cell 139, 485-498 (2009).

44. Atarashi, K. et al. Induction of colonic regulatory T cells by indigenous Clostridium species. Science 331, 337-341 (2011).

45. Palm, N.W. et al. Immunoglobulin a coating identifies colitogenic bacteria in inflammatory bowel disease. Cell 158, 1000-1010 (2014).

46. Kunii, J. et al. Commensal bacteria promote migration of mast cells into the intestine. Immunobiology 216, 692-697 (2011).

47. Ingersoll, M.A., Platt, A.M., Potteaux, S. \& Randolph, G.J. Monocyte trafficking in acute and chronic inflammation. Trends Immunol. 32, 470-477 (2011).

48. Xiong, H. \& Pamer, E.G. Monocytes and infection: modulator, messenger and effector. Immunobiology 220, 210-214 (2015).

49. Wang, B. et al. In vivo imaging implicates CCR2 $(+)$ monocytes as regulators of neutrophil recruitment during arthritis. Cell. Immunol. 278, 103-112 (2012).

50. Lefrancois, L. \& Lycke, N. Isolation of mouse small intestinal intraepithelial lymphocytes, Peyer's patch, and lamina propria cells. Curr. Prot. Immunol. Chapter 3, Unit 319 (2001). 